\title{
Comparison of the ligase chain reaction with cell culture for the diagnosis of Chlamydia trachomatis infection in women
}

\author{
G L Ridgway, G Mumtaz, A J Robinson, M Franchini, C Carder, J Burczak, H Lee
}

\begin{abstract}
Objective-To determine the sensitivity and specificity of ligase chain reaction (LCR) analysis of cervical and urine specimens from women compared with cell culture of cervical and urethral specimens for the diagnosis of genitourinary chlamydial infection.

Methods-Women $(n=624)$ attending the Genitourinary Medicine Clinic at University College London Hospitals, were enrolled. Patients who had received antibiotics within the previous two weeks were excluded. Specimens were obtained from the urethra and cervix for chlamydial culture, and from the cervix for LCR. A specimen of first void urine was also obtained for LCR. Discrepancies were resolved by direct immunofluorescence or a major outer membrane protein targeted LCR, or both.
\end{abstract}

Results-The prevalence of Chlamydia trachomatis in 600 patients, using an expanded standard of a positive cell culture or two confirmed positive non-culture tests, was $13 \cdot 2 \%$ (79/600). Cervical culture detected $68.4 \%$ and urethral culture $62 \%$ of all positive results compared with $81 \%$ detected by cervical LCR and $69 \%$ by urine LCR. Cervical and urethral culture combined detected $87 \cdot 3 \%$ whereas cervical and urine LCR combined detected $91.1 \%$ of positive cases. Specificity of LCR was $100 \%$ in the cervix and $99.8 \%$ in urine.

Conclusion-This study demonstrates that LCR analysis of cervical and urine specimens is a reliable method for the diagnosis of chlamydial genital infection in women. However, the study also demonstrates that no single test will detect all chlamydial infections. Conventional non-culture tests and cell culture may grossly underestimate the prevalence of chlamydial infection. LCR analysis of a cervical specimen was superior to conventional cell culture without blind passage as a single test for diagnosing chlamydial infection in women, followed by LCR of a urine specimen. (f Clin Pathol 1996;49:116-119)

Keywords: Chlamydia trachomatis, genital infection in women, ligase chain reaction.

The role of nucleic acid amplification technology in the routine diagnosis of infection is rapidly being established. Techniques include not only the polymerase chain reaction, but also the ligase chain reaction (LCR), self-sustained sequence replication, Ampliprobe, and $Q \beta$ replicase. ${ }^{1}$ Birkenmeyer and Armstrong ${ }^{2}$ reported preliminary studies on the use of LCR for the diagnosis of Neisseria gonorrhoeae infection, and Dille $e t a l^{\beta}$ reported on the detection of type strains of Chlamydia trachomatis with LCR, specifically targeting the cryptic plasmid.

One of the many problems in diagnosing genital infections is obtaining the sample. Testing urine for genital pathogens is very attractive as invasive sampling is not required, but urine is unsuitable for chlamydial culture. The advent of non-culture detection systems for chlamydiae has resulted in a re-appraisal of the use of urine, and this policy is finding increasing favour for the investigation of genital infection in men. ${ }^{4}$ Urine testing for chlamydial genital infection in women is more controversial, because infection may involve either the cervix or the urethra, or both sites. ${ }^{56}$ Initial reports showing that urine can be used in a LCR assay to detect chlamydial infection reliably in women as well as men are encouraging. ${ }^{7-9}$ Urine LCR can also be used to detect gonococcal genital infection reliably in women. ${ }^{10}$

One criticism of the chlamydial studies with LCR on women reported to date is that urine LCR was compared with cervical culture only. Cervical culture underestimates genital tract prevalence, whereas urine LCR potentially favours the detection of single site urethral infection. Here, we report a study comparing LCR analysis of cervical and urine specimens with cell culture of cervical and urethral specimens for the detection of chlamydial infection in women.

\section{Methods}

Women attending the Genitourinary Medicine Clinic at University College London Hospitals, who had not received antibiotics in the previous two weeks and who agreed to participate in the study, underwent a speculum examination of the vagina. The cervix was wiped with sterile gauze cotton-wool, and an endocervical swab taken and placed in 2SP transport medium for cell culture. A second endocervical swab was placed in LCR transport medium as supplied by the manufacturer. The order of obtaining cervical specimens was determined by a random number table. A third swab was gently introduced into the urethra and placed in 2SP medium as for the cervical specimen. Finally, $15-20 \mathrm{ml}$ of first void urine was obtained. Specimens for culture were either processed the same day having been held at $4^{\circ} \mathrm{C}$, or were

\footnotetext{
Correspondence to: Dr G L Ridgway.

Accepted for publication 18 October 1995

Abbott Laboratories, USA

J Burczak
} 
stored at $-70^{\circ} \mathrm{C}$ for up to 48 hours. Urine and cervical swabs for LCR were stored at $-70^{\circ} \mathrm{C}$ until tested. The study was approved by the University College London Hospitals' Ethics Committee.

Cell culture was carried out as described previously, using cycloheximide treated McCoy cells. ${ }^{11} \mathrm{~A}$ blind passage was performed on all samples. If the first pass culture was negative on iodine staining, the second pass was stained with a major outer membrane protein (MOMP) specific immunofluorescent monoclonal antibody (Syva Corp, Palo Alto, California, USA).

LCR was performed according to the manufacturer's instructions (Abbott Laboratories, Abbott Park, Illinois, USA). Urine samples were thawed, shaken to resuspend any solids, and $1 \mathrm{ml}$ transferred to a microcentrifuge tube. After centrifuging at $13000 \times g$ for 10 minutes, the supernatant fluid was removed and the deposit resuspended in $1 \mathrm{ml}$ urine resuspension buffer. The sample was then heated at $95^{\circ} \mathrm{C}$ for 15 minutes. Cervical specimens were heated at $95^{\circ} \mathrm{C}$ for 15 minutes and then on cooling, the swab was expressed against the side of the transport tube and discarded. A $100 \mu$ l aliquot of the prepared specimen (cervical or urine) was added to a unit dose tube. This latter contains all of the ingredients for the amplification step, including thermostable DNA ligase, thermostable DNA polymerase, NAD, magnesium, 2'-deoxycytidine 5'-triphosphate, $2^{\prime}$-deoxythymidine $5^{\prime}$-triphosphate, and an oligonucleotide probe set specific for the cryptic plasmid of $C$ trachomatis. ${ }^{3}$ Negative, positive and calibrator controls were similarly prepared. The unit dose tubes were subjected to 40 cycles of separation, annealing and ligation $\left(97^{\circ} \mathrm{C}\right.$ for one second, $55^{\circ} \mathrm{C}$ for one second, and $62^{\circ} \mathrm{C}$ for 50 seconds in each cycle) in a Perkin Elmer Cetus DNA Thermal Cycler. The product of the amplification step was assayed by microparticle enzyme immunoassay using the Abbott LCX analyser. Preparation of samples was carried out in an area separate from the thermocycler and LCX analyser. LCR inactivation reagent is automatically added to specimens after reading to prevent further contamination of subsequent samples.

Discrepancies were resolved by direct immunofluorescence (Microtrak, Syva) analysis of the relevant specimens. Samples found to be positive on plasmid LCR, but negative by cell culture and direct immunofluorescence were tested further using a MOMP based LCR.

Table 1 Sensitivity of detection of C trachomatis by LCR and cell culture with or without blind passage compared with total number of confirmed positive cases

\begin{tabular}{|c|c|c|c|c|}
\hline & \multicolumn{2}{|c|}{ Cell culture with blind passage } & \multicolumn{2}{|c|}{ Cell culture without blind passage } \\
\hline & $\begin{array}{l}\text { Number } \\
\text { detected } \\
(\text { total }=79)\end{array}$ & $\begin{array}{l}\text { Percentage } \\
\text { of total } \\
\text { positives }\end{array}$ & $\begin{array}{l}\text { Number } \\
\text { detected } \\
\text { (total }=79)\end{array}$ & $\begin{array}{l}\text { Percentage } \\
\text { of total } \\
\text { positives }\end{array}$ \\
\hline $\begin{array}{l}\text { Cervical culture } \\
\text { Urethral culture } \\
\text { Cervical and urethral culture } \\
\text { Cervical LCR } \\
\text { Urine LCR } \\
\text { Cervical and urine LCR }\end{array}$ & $\begin{array}{l}54 \\
49 \\
69 \\
64^{*} \\
55 \\
72^{*}\end{array}$ & $\begin{array}{l}68 \cdot 4 \\
62 \cdot 0 \\
87 \cdot 3 \\
81 \cdot 0 \\
69 \cdot 6 \\
91 \cdot 1\end{array}$ & $\begin{array}{l}44 \\
36 \\
57 \\
64^{*} \\
55 \\
72^{*}\end{array}$ & $\begin{array}{l}55 \cdot 7 \\
45 \cdot 6 \\
72 \cdot 2 \\
81 \cdot 0 \\
69 \cdot 6 \\
91 \cdot 1\end{array}$ \\
\hline
\end{tabular}

${ }^{*}$ Includes three specimens confirmed by MOMP LCR.
A confirmed positive was defined as either positive on cell culture or positive by two of the other tests (plasmid LCR and direct immunofluorescence, or plasmid LCR and MOMP LCR) at any site.

\section{Results}

Six hundred and twenty four women were entered into the study. Twenty four patients were excluded from further analysis for the following reasons. Two patients (one positive on urine and cervical LCR, and the other positive on cervical LCR only) had been positive for $C$ trachomatis elsewhere and had been treated recently. One patient had recently received antibiotics for another condition. Seven patients were inadvertently admitted to the study twice (the second episode is excluded). Eight patients did not have a urine specimen submitted for assay, one patient's urine assay tube broke, preventing testing, and another did not have a urethral specimen taken. Plasmid LCR specimens from the final 213 patients were analysed by Abbott Laboratories and four of the cervical specimens sent leaked in transit. Thus, complete results from 600 patients were available for analysis.

The prevalence of $C$ trachomatis on cell culture or LCR plus direct immunofluorescence, or both, was $12 \cdot 7 \%$ (76 of 600 ). A further three cervical specimens positive on plasmid LCR only were confirmed by MOMP LCR, and are considered true positive results, giving an overall prevalence of $13 \cdot 2 \%$ (79 of 600 ).

The sensitivities for the different tests and sample combinations are shown in table 1. Thus, culture of cervical and urethral specimens combined detected $87 \cdot 3 \%$ of all confirmed positive cases. However, it must be stressed that few laboratories perform culture with passage as a routine method to identify genital $C$ trachomatis, let alone take both a cervical and urethral specimen. The effect of only recording the results of first pass cell culture is also demonstrated in table 1. Whilst the results of LCR are unchanged, first pass culture of cervical and urethral specimens now detected only $72 \cdot 2 \%$ of all positive cases. Were only a single passage cervical culture to have been carried out, only $55.7 \%$ of all positive cases would have been detected. LCR analysis of cervical or urine specimens would have detected $81 \%$ and $69 \cdot 6 \%$ of all confirmed positive cases, respectively. When the results of LCR analysis of urine and cervical specimens were combined, $91 \cdot 1 \%$ of all confirmed positive cases were identified.

Table 2 compares the LCR results with the confirmed positive results. As expected, the specificity of the LCR test is very high. There were 25 confirmed positive results where the LCR analysis of cervical and urine specimens was discordant. The urine LCR was negative in 19 cases. In six of these a high negative was reported, which became a low positive on retesting three. In one case, where the cervical LCR was positive and all other tests negative, enzyme immunoassay and direct immunofluorescence on a cervical specimen obtained 
Table 2 Comparison of cervical and urine LCR results with confirmed $\mathrm{C}$ trachomatis positive results

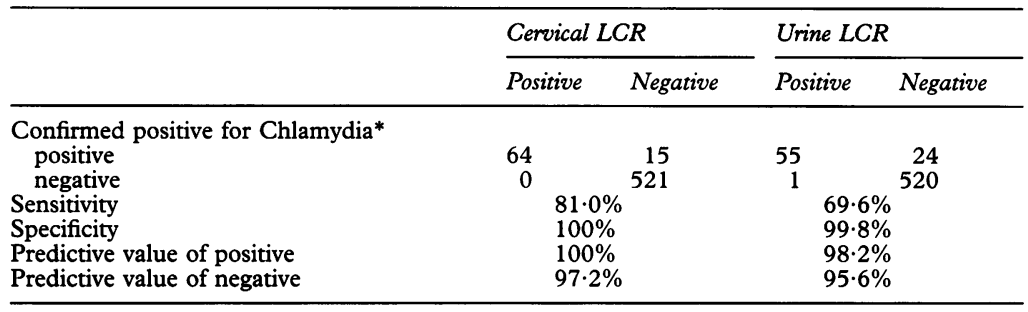

* Extended gold standard, defined as culture positive, or LCR plus direct immunofluorescence positive, or LCR confirmed by MOMP LCR at any site.

one week later were positive. In three cases the cervical LCR was the only positive test and retesting using a MOMP based LCR confirmed that they were indeed positive. In one of these cases the urine was also positive with the MOMP based LCR. Six specimens were positive on urine LCR and negative on cervical LCR. In five of these only the urethral culture was positive, whereas culture and direct immunofluorescence analysis of the cervical specimen were negative. In the sixth case the cervical LCR reading was a high negative and culture of the cervical swab was positive. We have no explanation for the single false positive urine LCR result; repeat testing of the pellet and another aliquot of the same specimen was negative.

Table 3 demonstrates the sensitivity, specificity, and positive and negative predictive values of a routine first pass cell culture compared with our extended method. Of note is the apparent low sensitivity of first pass cell culture when compared with the extended method used in this study.

We elected to record cell culture specimens that were overgrown with Candida sp. or toxic to cell culture as negative, because we had other means (LCR and direct immunofluorescence) for resolving any unexpected, discrepant results. Toxic or contaminated cell cultures cannot be recorded routinely as negative, as it is not possible to exclude the presence of $C$ trachomatis without further tests. In this study cultures (cervical and/or urethral) from 113 patients $(18.8 \%)$ would have been indeterminate because of overgrowth by Candida sp. Only two specimens toxic to cell culture were LCR positive (both from the cervix), and in both cases the direct immunofluorescence analysis confirmed infection in the cervix. In one of these patients the urine LCR was also positive.

Table 3 Comparison of cervical and urethral first pass cell culture results with confirmed $\mathrm{C}$ trachomatis positive results

\begin{tabular}{|c|c|c|c|c|}
\hline & \multicolumn{2}{|c|}{ Cervical culture } & \multicolumn{2}{|c|}{ Urethral culture } \\
\hline & Positive & Negative & Positive & Negative \\
\hline $\begin{array}{l}\text { Confirmed positive for Chlamydia* } \\
\text { positive } \\
\text { negative }\end{array}$ & $\begin{array}{r}44 \\
0\end{array}$ & $\begin{array}{r}35 \\
521\end{array}$ & $\begin{array}{r}36 \\
0\end{array}$ & $\begin{array}{r}43 \\
521\end{array}$ \\
\hline $\begin{array}{l}\text { Sensitivity } \\
\text { Specificity } \\
\text { Predictive value of positive } \\
\text { Predictive value of negative }\end{array}$ & \multicolumn{2}{|c|}{$\begin{array}{l}55 \cdot 7 \% \\
100 \% \\
100 \% \\
93 \cdot 7 \%\end{array}$} & \multicolumn{2}{|c|}{$\begin{array}{l}45 \cdot 6 \% \\
100 \% \\
100 \% \\
92 \cdot 4 \%\end{array}$} \\
\hline
\end{tabular}

* Extended gold standard, defined as culture positive, or LCR plus direct immunofluorescence positive, or LCR confirmed by MOMP LCR at any site.

\section{Discussion}

This study set out to answer two important questions. Firstly, to determine the overall sensitivity of LCR analysis of urine or cervical specimens when compared with a cell culture system that included a single blind passage, backed up by a direct monoclonal antibody immunofluorescence test; and secondly, to compare how LCR tests on urine and cervical specimens compared with a routine cell culture diagnostic system without passage. We cultured specimens from the urethra and cervix in all cases to ensure maximum yield of positive isolates.

It is well recognised that cell culture is far from being the gold standard for chlamydial diagnosis, ${ }^{12}$ and that in women investigation of specimens from only one site will underestimate the prevalence of chlamydial genital infection..$^{56}$ The use of urine samples from women has not been considered feasible previously because of the predominately cervical site of chlamydial infection. Hay et at ${ }^{6}$ recently demonstrated that direct immunofluorescence analysis of the centrifuged deposit of a first pass urine from women was $80 \%$ sensitive compared with a cervical smear sensitivity of $70 \%$ for diagnosing chlamydial genital infection, using the combined results of centrifuged urine deposit, urethral smear, cervical smear and centrifuged cervical swab deposit as the standard.

Chernesky et al studied 447 women, and found the sensitivity of LCR on first void urine to be $96 \cdot 3 \%$, compared with $55 \cdot 6 \%$ for cervical culture (with single blind passage) and $78.3 \%$ for cervical enzyme immunoassay (confirmed Chlamydiazyme test (Abbott Laboratories)). A four centre study extended these findings, analysing cervical specimens and first void urine samples from 1937 women by culture and LCR assay, respectively. ${ }^{8}$ Urine LCR was $93 \cdot 8 \%$ sensitive compared with a cell culture sensitivity of $65 \cdot 0 \%$. Bassiri et al compared urine LCR with cervical cell culture and enzyme immunoassay of urine (IDEIA III, Novonordisk Diagnostics, Berkshire, UK). They studied 447 women, and found sensitivities of $56.3 \%$ on cell culture, $18.8 \%$ on urine enzyme immunoassay and $87.5 \%$ on urine LCR. Thus, all three studies have found that urine LCR was much superior to cervical cell culture. For urine LCR specificity ranged between 99.9 and $100 \%$. However, these studies did not investigate culture specimens from the urethra, nor was LCR carried out on cervical specimens. Cervical culture would therefore underestimate total genitourinary tract infection, and may favourably bias the urinary LCR results. Comparison of urethral and cervical culture with urine or cervical LCR has demonstrated that LCR analysis of urine or cervical specimens is more sensitive than a single cervical culture without passage, such as would be carried out routinely in a centre using cell culture for diagnosis.

Of concern is that the quoted sensitivity of commercially available enzyme immunoassays is within the range $70-90 \%$ compared with cell culture (often without blind passage). ${ }^{13}$ The sensitivity of cervical cell culture in this and the other reviewed studies ${ }^{7-9}$ was between $55 \cdot 6$ 
and $68.4 \%$. It is therefore apparent that current routine procedures in the field are liable to be grossly underestimating the prevalence of chlamydial genital infection in women.

We have demonstrated that LCR is consistently more reliable than cervical or urethral cell culture alone, either with or without blind passage. Analysis of urine or cervical specimens by LCR assay was of equivalent sensitivity to combined cervical and urethral cell culture without passage. Indeed, for a cervical culture without passage LCR was superior $(72 \cdot 2 \%$ and $81.0 \%$, respectively). The most reliable tests in this study were either a combined cervical and urethral multiple passage cell culture, or a combined LCR analysis of cervical and urine samples. Neither of these latter options is feasible in the routine diagnostic setting, and it should be noted that $12 \cdot 7 \%$ and $8 \cdot 9 \%$, respectively, of confirmed positive results were missed by the combined tests.

We conclude that detection of genital $C$ trachomatis infection in women by use of the LCR test on cervical or urine specimens is a convenient and more reliable substitute for cell culture in a diagnostic setting.

1 Birkenmeyer L, Mushahwar IK. DNA probe amplification methods. F Virol Methods 1991;35:117-26.

2 Birkenmeyer L, Armstrong A. Preliminary evaluation of the ligase chain reaction for specific detection of Neisseria gonorrhoeae. $\mathcal{f}$ Clin Microbiol 1992;30:3089-94.
3 Dille BJ, Butzen CC, Birkenmeyer L. Amplification of C. trachomatis DNA by ligase chain reaction. $\mathcal{F}$ Clin Microbiol 1993;31:729-31.

4 Ridgway GL, Taylor-Robinson D. Curent problems in microbiology: I Chlamydial infections: Which laboratory test? F Clin Pathol 1991;44:1-5.

5 Jones RB, Katz BP, Van der Pol B, Caine VA, Batteiger BE, Newhall VWJ. Effect of blind passage and multiple sampling on recovery of Chlamydia trachomatis from sampling on recovery of Chlamydia trachomatis from

uronital specimens. f Clin Microbiol 1986;24:1029-33. Tay PE, Thomas BJ, Horner PJ, Macleod E, Renton AM, the more you look, the more you find. Genitourin Med the more you lo

7 Chernesky M, Jang D, Lee H, Burczak JD, Hu H, Sellors $\mathrm{J}$, et al. Diagnosis of Chlamydia trachomatis infections in men and women by testing first void urine by ligase chain reaction. 7 Clin Microbiol 1994;32:2682-5.

8 Lee H, Chernesky M, Schachter J, Burczak JD, Andrews WW, Muldoon S, et al. Diagnosis of Chlamydia trachomatis genitourinary infection in women by ligase chain matis genitourinary infection in women by ligase

9 Bassiri M, Hu HY, Domeika MA, Burczak J, Svensson $\mathrm{L}-\mathrm{O}$, Lee $\mathrm{HH}$, et al. Detection of Chlamydia trachomatis in urine specimens from women by ligase chain reaction. $f$ Clin Microbiol 1995;33:898-900.

10 Smith KR, Ching S, Lee H, Ohhashi Y, Hu H-Y, Fisher $\mathrm{HC}$, et al. Evaluation of ligase chain reaction for use with urine for identification of Neisseria gonorrhoeae in females attending a sexually transmitted disease clinic. $f$ Clin Micmbiol 1995;33:455-7.

11 Mumtaz G, Clark S, Ridgway GL, Miller CJ, Johal B, Allason-Jones E. Comparison of an enzyme immunoassay with cell culture for the detection of genital chlamydial infection in high and low risk populations. Genitourin Med 1993;69:119-22.

12 Schachter J, Stamm W, Chernesky M, Hook EW, Jones RB, Judson FN, et al. Nonculture tests for chlamydial infection. What does the package insert mean, and will it mean the same thing tomorrow? Sex Transm Dis 1992;16:243-4.

13 Schachter J. Diagnosis of Chlamydia trachomatis infection. In: Orfila J, Byrne GI, Chernesky MA, Grayston JT, Jones $\mathrm{RB}$, Ridgway GL, et al, eds. Chlamydial infections. Bologna: Società Editrice Esculapio, 1994:293-302. 